\title{
Predicting Relapse to Alcohol and Drug Abuse via Quantitative Electroencephalography
}

\author{
Lance O. Bauer, Ph.D.
}

A sensitive and specific screening test that would identify the subset of substance-abusing patients at highest risk for relapse would constitute an important advance for treatment planning. This study examined the relative value of quantitative electroencephalography as a rapid, inexpensive, and noninvasive measure of relapse potential. The subjects were 107 substance-dependent patients enrolled in residential treatment programs. All were unmedicated and free of the complicating effects of major medical and neurological disorders. Structured clinical interview data and a 5-minute recording of the resting, eyes-closed electroencephalogram were obtained after patients had verifiably maintained abstinence for 1-5 months. Patients were then monitored for relapse or successful abstinence by research staff for an ensuing 6-month period. ANCOVAs of EEG power spectral density within pre-defined frequency bands revealed an enhanced amount of high frequency (19.5-39.8 Hz) $\beta$ activity among the 48 patients who later relapsed compared to both 59 patients who maintained abstinence and 22 additional subjects with no history of substance dependence. Importantly, in subsequent logistic regression analyses, fast $\beta$ power was found to be superior to severity of illness, depression level, and childhood conduct problems in predicting relapse. With fast $\beta$ power as the sole predictor, the sensitivity, specificity, and positive and negative predictive value parameters for discriminating outcomes were $0.61,0.85,0.75$, and 0.74 , respectively. Additional ANCOVAs revealed that the EEG difference between relapse-prone and abstinence-prone groups was related to the interaction of two premorbid factors, viz., childhood Conduct Disorder and paternal alcoholism. The enhancement of fast $\beta$ electroencephalographic activity in patients who will later relapse most likely originates from a premorbid and subtle dysfunction involving frontal brain regions.

[Neuropsychopharmacology 25:332-340, 2001] (C) 2001 American College of Neuropsychopharmacology. Published by Elsevier Science Inc.
KEY WORDS: Electroencephalography; Relapse; Alcohol; Cocaine; Heroin; Alcoholism; Drug Abuse; Substance related disorders; Recurrence; Frontal Cortex

Relapse rates following treatment for substance dependence are remarkably high, and efforts to address this unfortunate reality are becoming an integral part of treatment

From the Department of Psychiatry, MC-2103, University of Connecticut Health Center, Farmington, CT 06030, Tel.: 860-679-4154, Fax: 860-679-4077, E-mail: bauer@psychiatry. uchc.edu

Address correspondence to: Lance Bauer, Ph.D., Neural Dynamics Laboratory, University of Connecticut School of Medicine, Farmington, CT 06030-2103

Received July 24, 2000; revised November 2, 2000; accepted February 2, 2001.

Online publication: 2/7/01 at www.org/citations/Npp02070177.
(Daley and Marlatt 1997). In outcome studies of alcoholics, for example, approximately $65-70 \%$ of patients have been found to relapse within one year of treatment, with the majority of these patients relapsing within less than three months (Hunt et al. 1971; Emrick 1974; Miller and Hester 1986). In outcome studies of drug- or polysubstance-dependent patients, relapse rates following treatment are similar to, if not greater than, those found for patients solely dependent on alcohol (Emrick 1974; McKay et al. 1999).

A number of investigators have focused on analyzing the multiple variables associated with relapse in an attempt to develop screening tools that might identify subpopulations most in need of relapse-prevention treatment. A number of risk factors have been identified. More severe levels of alcohol or drug dependence 
have, for example, been associated with an increased likelihood of relapse (Booth et al. 1991; Kampman et al. 1998; Langenbucher et al. 1996; McLellan et al. 1994; Winterer et al. 1998). In such studies, severity of dependence has been quantified using scores on either alcohol or drug use problem scales, DSM symptom counts, or the number of previous hospitalizations.

The presence of an additional, comorbid psychiatric disorder is likewise associated with an increased risk for relapse. One such disorder is the presence of severe conduct problems both before and after age 15, i.e., a conjoint diagnosis of Conduct Disorder (CD) and Antisocial Personality Disorder (ASPD) (Hesselbrock 1991; Leal et al. 1994; Woody et al. 1984, 1985). Comorbid depression also appears to promote relapse. However, the association between comorbid depression and treatment outcome is complex, and appears to depend upon the nature of the treatment, the gender and age composition of the patient sample, and the preferred drug of abuse (Hesselbrock 1991; Bobo et al. 1998; Brown et al. 1998; Hodgins et al. 1999; Kranzler et al. 1996; Rounsaville et al. 1986; Sellman and Joyce 1996; Ziedonis and Kosten 1991).

Although the above-mentioned clinical studies have demonstrated an association between greater illness severity, psychiatric comorbidity, and relapse, the association has not been replicated in other studies. Furthermore, when these same variables have been entered into more rigorous analyses (e.g., logistic regression or discriminant function) designed to predict the outcome of individual cases, the resulting sensitivity and specificity in prediction have been less than ideal. In part, the limited power of CD/ASPD, depression, and illness severity for specifying outcome might be attributed to inaccuracy in the patient's recall of his/her history and severity of substance abuse as well as imprecision in the diagnosis of comorbid disorders. Accordingly, the addition of an objective measure, which is less influenced by the reliabilities and validities of the patient's report and the clinical diagnosis, might markedly improve our ability to forecast outcome and plan appropriate preventive measures.

Several recent studies employing objective and quantitative electroencephalographic (EEG) techniques have already demonstrated an association between the amount of $\beta$ (i.e. $>13 \mathrm{~Hz}$ ) activity in the spontaneous electroencephalogram and relapse to alcohol or cocaine abuse. Our initial report on this topic (Bauer 1994) described a study of 17 alcohol-dependent inpatients and 14 healthy, nonalcoholic volunteers from the community. The patients were tested after 7 and 14 days of verified abstinence. The control group was also repeatedly tested to control for familiarization effects. All of the participants were medication-free at the time of testing and were screened to exclude individuals with other drug dependence, seizures, and similar factors that would complicate their EEGs. Patients were followed for a 3-month period after leaving treatment. Via detection procedures which included self-reports, weekly breathalyzer tests, and collateral informants, the patient group was divided into subgroups defined by the presence versus absence of any alcohol use during the 3-month post-treatment period. Differences in baseline EEG spectral power, and in autonomic, demographic, and other pre-treatment characteristics were tested between the Relapse-prone and Abstinence-prone patient groups, and the nonalcoholic control group.

The results showed that patients with unfavorable versus favorable outcomes were similar in demographic and clinical characteristics. In the face of these similarities, EEG activity alone significantly differentiated the groups: Relapse-prone patients exhibited more high frequency $\beta$ activity in their EEGs than Abstinence-prone patients. This suggestion of heightened central nervous system arousal associated with future relapse was convergently validated by the demonstration of corresponding elevations in cardiac output and rate within the Relapse-prone group.

In 1998, a group from Germany (Winterer et al. 1998) replicated our 1994 findings using 38 alcohol-dependent patients studied after seven days of abstinence. Their analyses of demographic and substance use characteristics also revealed relatively few differences between the Abstinence-prone and Relapse-prone groups. When measures of EEG activity were entered into a discriminant function analysis, the sensitivity for predicting relapse was about $88 \%$. Abstinent outcomes were correctly identified for $92 \%$ of the cases. The overall classification accuracy of approximately $90 \%$ was greater than the $59 \%$ accuracy associated with only clinical measures.

In 1999, a research group from New York University (Prichep et al. 1999) extended the idea of relating baseline EEG activity to outcome in a group of cocainedependent patients enrolled in a residential treatment program. Their approach to the analysis was somewhat different. For example, in the NYU study, retention in treatment was the criterion variable, not relapse per se. These investigators also assigned their 35 patients to groups not on the basis of treatment outcome, but on the basis of a cluster analysis of several EEG variables. The relationships between the empirically-derived EEG clusters and several demographic and substance use variables were then examined. There were no significant differences between the EEG clusters except for the membership's average length of stay in residential treatment. One cluster of patients, which was characterized by a shorter length of stay in treatment in comparison to the other cluster, was also characterized by a relative abundance of EEG $\beta$ activity. When viewed in context, the NYU study is interesting because it suggests that the association between enhanced EEG $\beta$ activity and negative outcome generalizes to another substance-abusing population (viz. cocaine abusers) and to 
an alternate operational definition of a negative outcome (viz. a shorter length of stay in treatment).

The present study was designed to extend previous EEG studies of relapse prediction in five key areas: (1) It utilized a larger number of patients $(\mathrm{N}=107)$ than previous EEG studies and therefore provided adequate statistical power and the opportunity for subgroup analyses. (2) It formally determined whether the value of EEG activity as a predictor of relapse exceeds that of other variables which are more easily and less expensively measured. (3) The present study formally tested the generality of EEG as a relapse predictor across four different types of substance dependence. (4) The present study utilized collateral informants and frequent (1-2x/week), unannounced urine and breath assays as the method for detecting relapse. (5) And, finally, the present study examined whether EEG methods are informative in a residential treatment population that has already demonstrated at least one month of successful abstinence.

\section{METHODS}

\section{Patient Recruitment and Screening}

Data were obtained from 107 patients carefully selected from residential substance abuse treatment programs in the Hartford, Connecticut, area. As a control, an additional 22 subjects were recruited. The latter group of subjects had no history of substance dependence.

All of the subjects were healthy. None was taking prescribed or non-prescribed medications. Candidates were also excluded if they had a history of seizures, head injury, schizophrenia, major affective disorder, mental retardation, or other significant medical disorders, including cardiovascular, hepatic, immunologic, or renal disease. Medical and psychiatric histories were evaluated using structured interviews, viz., the SSAGA (Bucholz et al. 1994) for DSM-IIIR and the Addiction Severity Index (McLellan et al. 1980), administered by a trained research assistant. The interviews were audiotaped and monitored by the author to verify their consistency and validity. Additional demographic, medical, psychological, and drug use information was garnered from medical records and questionnaires, viz., Michigan Alcoholism Screening Test (MAST; Selzer 1971), Drug Abuse Screening Test (DAST-10; Skinner 1982), Spielberger State-Trait Anxiety Inventory (STAI; Spielberger 1983), Beck Depression Inventory (BDI; Beck et al. 1961), and the Shipley Institute of Living Scale (SILS; Shipley 1940).

At the time of the EEG assessment, patients had been abstinent for minimum of one month and maximum of five months. Following the EEG assessment and for a subsequent 6-month period, patients were visited frequently (i.e. 1-2x/week) and unpredictably for the pur- pose of detecting relapse or continued abstinence via urine (EZ Screen, Editek Inc.) and breath screening. Interviews with patients and informants were also conducted at these times. Patients were assigned to the Relapse-prone group if they used alcohol, cocaine, opiates, or benzodiazepines on one or more occassions during a 6-month tracking period. Patients were assigned to the Abstinence-prone group if they demonstrably maintained abstinence from these substances over the 6-month tracking period. Prior to their formal enrollment in the study, patients and controls signed an approved informed consent agreement describing the research procedures and the study's risks and benefits.

\section{Acquisition and Reduction of EEG Data}

EEG activity was amplified (gain $=10 \mathrm{~K}$, bandpass = $0.1-40 \mathrm{~Hz}$ ) and digitized at $100 \mathrm{~Hz}$ over a 5-minute period under eyes-closed conditions. Fifteen electrode sites, arranged in the International 10/20 System, were sampled. EEG epochs of $1.28 \mathrm{sec}$ duration were detrended, baseline-corrected, and cosine tapered. The epochs were edited for EMG/movement (peak-to-peak deflection > 50 microvolts), lead sway and A-D converter overflow artifacts, and mathematically corrected for eye movement artifact. The accepted EEG epochs were then submitted to a Fast Fourier Transform which computed the power (in $\mu \mathrm{V}^{2}$ ) of the EEG in $0.78 \mathrm{~Hz}$ steps over a total range of $1.5-40 \mathrm{~Hz}$. The power spectra were averaged over epochs. Estimates of power within the $\delta(1.5-3.2$ $\mathrm{Hz}), \theta(3.8-7.02 \mathrm{~Hz}), \alpha(7.8-12.5 \mathrm{~Hz})$, and slow $(13.2-$ $18.75 \mathrm{~Hz})$ and fast $(19.5-39.8 \mathrm{~Hz}) \beta$ frequency bands were expressed as a proportion of total power across the entire frequency range. For the purpose of maximizing reliability and minimizing noise, these relative power estimates for each frequency band were averaged across the 15 electrode sites in all but one of the analyses (see below).

\section{Data Analysis}

Demographic, psychological, and drug use differences between the Abstinence-prone and Relapse-prone patient groups and the Non-Dependent Control group were evaluated using one-way ANOVA for continuous variables and Pearson's Chi-Square test for categorical variables.

The strategy for evaluating the relationship between EEG activity and outcome proceeded in several stages:

The first stage in the analysis was a set of five 1-way ANOVAs comparing the three subject groups (Abstinence-prone, Relapse-prone, Control). To eliminate the potential complication of subtle and confounded group differences in illness severity, alcohol (MAST), drug use (DAST-10), and depression (BDI) severity scores were entered as covariates. Separate ANCOVAs were per- 
formed for relative power in each of the five preselected, nonoverlapping frequency bands. Tukey post hoc tests were used to determine the source of significant differences suggested by the ANCOVA.

Another ANCOVA was performed focusing only on the EEG frequency that revealed group differences in the prior analysis. This analysis was designed to examine the legitimacy of combining data across electrode sites. The ANCOVA included electrode site and outcome as within- and between-subjects factors, respectively. The effects of illness severity were again controlled by utilizing MAST, DAST-10, and BDI scores as covariates. The control group was omitted from this and all subsequent analyses.

The third analysis involved an assignment of the 107 patients to one of four groups defined by their DSM-IIIR substance dependence diagnosis (alcohol, cocaine, alcohol and cocaine, or opioid/polydrug dependence) during the 12 months preceding admission to residential treatment. This analysis did not utilize covariates. It was structured as a 4 (diagnostic group) by 2 (outcome) ANOVA, with EEG fast $\beta$ power as the dependent variable.

The fourth stage in the analysis included childhood Conduct Disorder (CD) and paternal alcoholism, along with outcome, as independent variables in a single $2 \times$ $2 \times 2$ ANCOVA.

The final two analyses utilized stepwise logistic regression for the purpose of evaluating the predictive value of clinical measures alone versus the combination of clinical measures and EEG fast $\beta$ power. Cohen's Kappa (k) statistic was used to formally evaluate the agreement between actual and predicted outcomes. F-to-enter statistics and the respective significance lev- els provided an estimate of the relative value of various predictors. Predictive accuracy was summarized using standard descriptors:

- Sensitivity $=$ Proportion of true positives (relapse) who test positive [\# true positive/ (\# true positive + \# false negative)].

- Specificity = Proportion of true negatives (abstinent) who test negative [\# true negative/ (\# false positive + \# true negative)].

- Positive Predictive Value = Proportion of test positives who are in fact positive [\# true positive/(\# true positive + \# false positive)].

- Negative Predictive Value $=$ Proportion of test negatives who are in fact negative [\# true negative/(\# false negative $+\#$ true negative)].

\section{RESULTS}

The demographic, psychological, and substance use characteristics of the Abstinence-prone, Relapse-prone, and Non-Dependent Control groups are shown in Table 1. On average, subjects were 34.9 years of age. Approximately $67 \%$ of the patients were male. Approximately $45 \%$ were Caucasian.

The two patient groups reported higher scores than the Non-Dependent Control group on depression, trait anxiety, and alcohol and drug problem scales. However, the patient groups did not differ from one another on these scales. Both patient groups had been abstinent for a similar period (approx. three months) at the time of baseline EEG data collection.

Table 1. Demographic and Baseline Psychological and Substance Use Characteristics of Subject Groups

\begin{tabular}{|c|c|c|c|c|}
\hline & Abstinent & Relapse & Non-Dependent & \\
\hline & $n=59$ & $n=48$ & $\mathrm{n}=\mathbf{2 2}$ & Test Result \\
\hline Age in yrs $\pm S D$ & $35.4 \pm 6.6$ & $33.9 \pm 6.4$ & $35.6 \pm 6.1$ & N.S. \\
\hline$\%$ Male & 62 & 76 & 59 & N.S. \\
\hline$\%$ Caucasian & 44 & 45 & 45 & N.S. \\
\hline IQ (SILS) & $94.9 \pm 10.9$ & $88.3 \pm 14.2$ & $103.9 \pm 12.2$ & $\begin{array}{c}\mathrm{F}=10.7 \mathrm{p}<0.05 \\
(\mathrm{AB} \& \mathrm{REL})<\mathrm{NON}\end{array}$ \\
\hline Depression (BDI) & $9.9 \pm 6.3$ & $9.2 \pm 6.2$ & $4.1 \pm 3.8$ & $\begin{array}{c}\mathrm{F}=7.6 \mathrm{p}<0.05 \\
(\mathrm{AB} \& \mathrm{REL})>\mathrm{NON}\end{array}$ \\
\hline Trait Anxiety (STAI) & $44.2 \pm 9.1$ & $45.6 \pm 9.2$ & $35.1 \pm 10.1$ & $\begin{array}{c}\mathrm{F}=9.9 \mathrm{p}<0.05 \\
(\mathrm{AB} \& \mathrm{REL})>\mathrm{NON}\end{array}$ \\
\hline $\begin{array}{l}\text { \# Alcohol Problems } \\
\text { (MAST) }\end{array}$ & $11.7 \pm 7$ & $13.6 \pm 7$ & $2.2 \pm 1.8$ & $\begin{array}{c}\mathrm{F}=22.4 \mathrm{p}<0.05 \\
(\mathrm{AB} \& \mathrm{REL})>\mathrm{NON}\end{array}$ \\
\hline $\begin{array}{l}\text { \# Drug Problems } \\
\text { (DAST-10) }\end{array}$ & $7.0 \pm 2.5$ & $6.9 \pm 2.9$ & $0.2 \pm 0.5$ & $\begin{array}{c}\mathrm{F}=66.1 \mathrm{p}<0.05 \\
(\mathrm{AB} \& \mathrm{REL})>\mathrm{NON}\end{array}$ \\
\hline $\begin{array}{c}\text { Months Abstinent @ } \\
\text { EEG Evaluation }\end{array}$ & $2.9 \pm 1.5$ & $2.7 \pm 1.2$ & NA & N.S. \\
\hline $\begin{array}{l}\% \text { Family History of } \\
\text { Alcoholism }\end{array}$ & 69.6 & 55 & 33 & N.S. \\
\hline $\begin{array}{l}\text { \% DSM-IIIR } \\
\text { Conduct Disorder }\end{array}$ & 28.8 & 52.1 & 0 & $\begin{array}{c}\mathrm{x}^{2}=16.3 \mathrm{p}<0.05 \\
\mathrm{REL}>\mathrm{AB}>\mathrm{NON}\end{array}$ \\
\hline
\end{tabular}


Only two background measures differentiated between the Relapse-prone and Abstinence-prone groups. The Relapse-prone group was comprised of a higher percentage of members meeting DSM-IIIR diagnostic criteria for childhood Conduct Disorder. In addition, Relapse-prone patients exhibited a significantly lower IQ score on the SILS than the other group.

The overall effect of subject group on relative EEG power within the five preselected frequency bands was evaluated using five separate one-way ANCOVAs. Figure 1 shows that power in the high frequency $\beta$ band significantly differentiated the groups $(\mathrm{F}=3.6, p=.03)$. Tukey post-hoc tests showed that Relapse-prone patients exhibited significantly more high frequency $\beta$ activity than the other two groups.

A topographic analysis (Electrode Site $\times$ Outcome Group ANCOVA) of fast $\beta$ power for the Abstinenceprone and Relapse-prone groups revealed that power was maximal (Electrode Site: $\mathrm{F}=5.26, p=.009$ ) in regions along and anterior to the central sulcus. The scalp distribution was similar in both groups (Electrode Site $\times$ Outcome Group: $\mathrm{F}=0.73, p=.46$ ).

To further localize the anatomical source of EEG fast $\beta$ power, we submitted group-averaged fast $\beta$ power for each electrode site to Current Source Density analysis and constrained the search for sources via the Boundary Element Method. CSD analysis is a state-ofthe-art method which attempts to find a distributed solution for source localization. CSD differs from dipole modeling algorithms which can make unrealistic as- sumptions about the number of likely generators and their size or orientation. The Boundary Element Method represents a significant enhancement in localization algorithms because it constrains the search for sources according to actual skin, skull, and cortical depths and shapes. It is, in fact, based on a structural MRI as the model, as opposed to non-BEM methods which assume that the head and brain form concentric spheres. As one can see in Figure 2, the Minimum Norm Least Squares CSD/BEM algorithm identified the most anterior regions of the frontal brain as the likely source of fast $\beta$ power.

A separate analysis of the data asked whether the abnormal elevation in EEG fast $\beta$ power associated with future relapse varied with the preferred substance of abuse. For this analysis, patients were assigned to one of four mutually exclusive groups, viz., alcohol-dependent $(n=21)$, cocaine-dependent $(n=28)$, alcohol- and cocaine-dependent $(n=34)$, or opioid/polydrugdependent $(\mathrm{n}=24)$, using DSM-IIIR criteria. The analysis revealed significantly $(\mathrm{F}=3.2, p=.02)$ greater levels of fast $\beta$ activity in the cocaine- and alcohol-dependent, alcohol-dependent and cocaine-dependent groups relative to the opioid/polydrug dependent group. Yet, the interaction of outcome and type of substance dependence was not significant $(\mathrm{F}=0.29, p=.83)$. Furthermore, tests of the effects of outcome were significant at $p<.05$ for all four categories of dependence.

Since the abnormal elevation in $\beta$ power in Relapseprone patients is not related to their preferred drug of
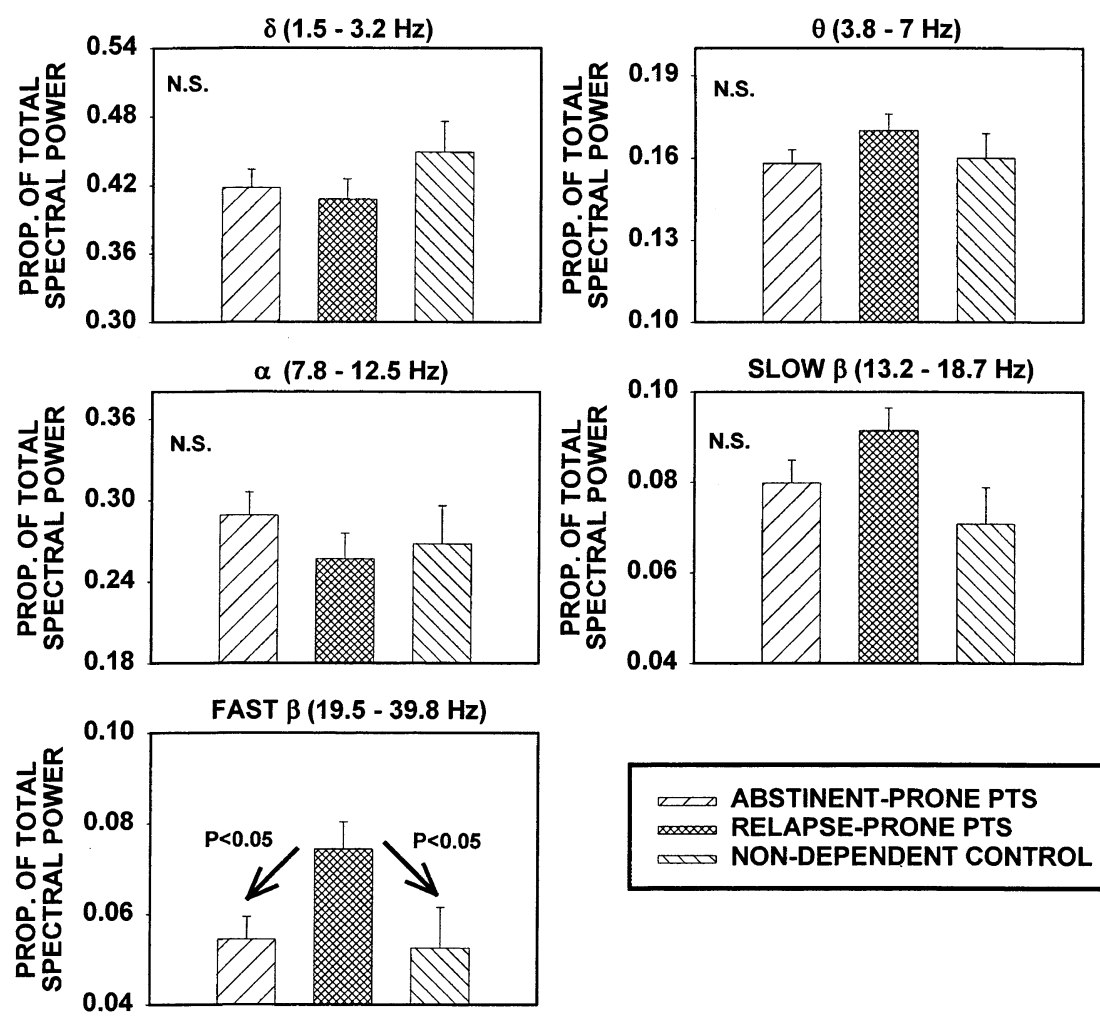

Figure 1. Relative power ( $+1 \mathrm{SE})$ in the $\delta, \theta, \alpha$, and slow and fast $\beta$ EEG frequency bands as a function of group (Relapseprone, Abstinence-prone, or Non-dependent Control). 


\section{CSD/BEM TOPOGRAPHIC MAP OF FAST $\beta$ POWER}

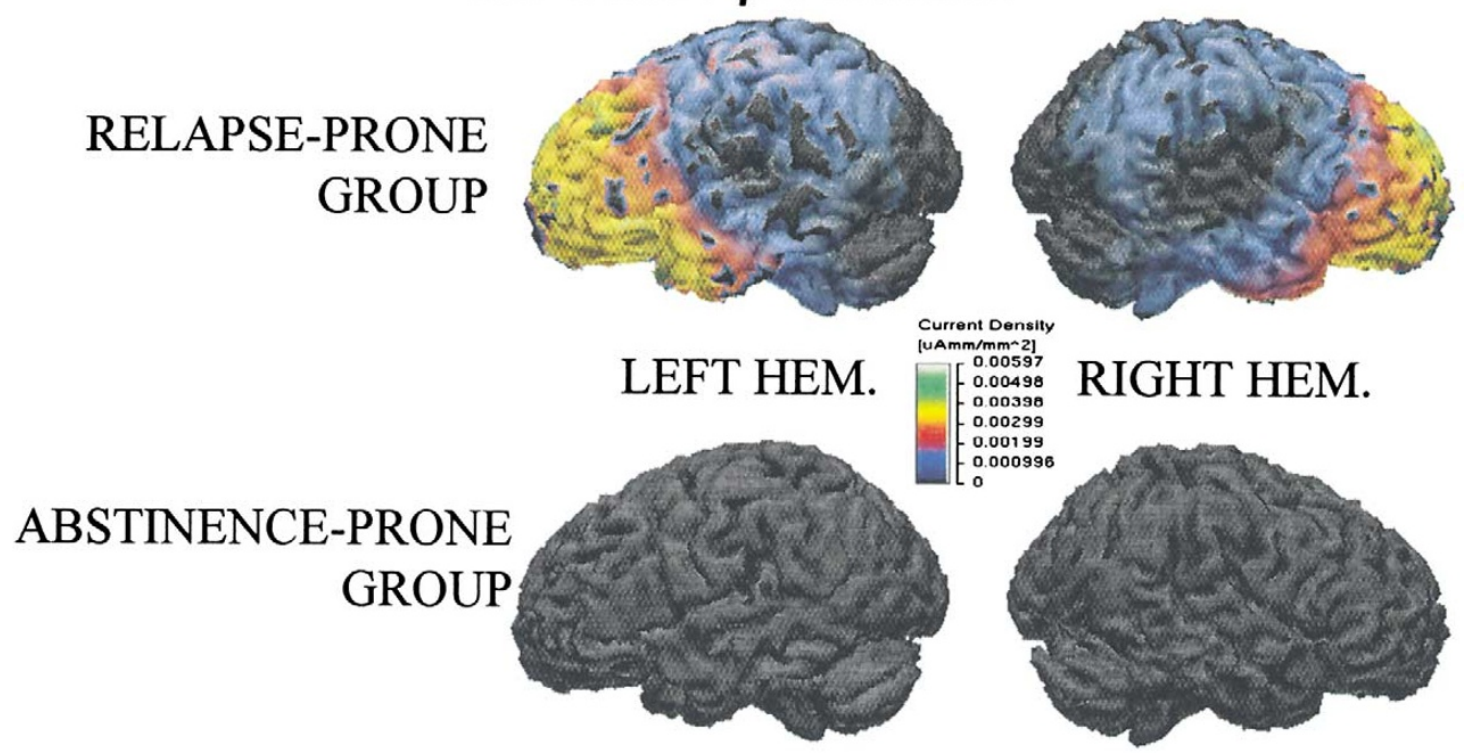

Figure 2. Topographic map of current source density distribution of fast $\beta$ power for the Relapse-prone and Abstinenceprone groups. The yellow region defines an area characterized by maximal CSD.

abuse, might it be related to a premorbid factor that is common across alcohol, cocaine, and opioid dependence? A 3-way ANOVA was used to test for interactions between two known risk factors, namely a family history of alcoholism (Cotton 1979) and DSM-IIIR Conduct Disorder (Robins 1966), and outcome. The interaction of these three variables was significant $(\mathrm{F}=8.02, p=$ .006; Figure 3), suggesting that the neurophysiological abnormality associated with relapse is related to the same neurophysiological abnormality that accompanies increased risk for the initial onset of alcoholism and drug abuse.

We then asked the more important question regarding the relative value of clinical, drug use, and EEG predictors of relapse. The first logistic regression (Table 2) included the number of alcohol and drug abuse problems, and the four combinations of the paternal alcoholism by Conduct Disorder factorial. The resulting accuracy in predicting outcome using these characteristics was no better than chance. The average classification accuracy was only $56.5 \%$. The associated test for agreement between actual and predicted outcomes was not significant (Cohen's $\kappa=0.1, p=$ n.s.).

The second logistic regression included these same variables along with fast $\beta$ power. With this change in input parameters, the accuracy in predicting outcome improved from $56.5 \%$ to $74.3 \%$ - a better than chance level. The statistics evaluating the level of agreement between actual and predicted outcomes also improved (Cohen's $\kappa=0.45, p<.0001$; Sensitivity $=0.61$, Speci- ficity $=0.85$, Positive Predictive Value $=0.75$; Negative Predictive Value $=0.73$ ). Most importantly, though, fast $\beta$ power far outweighed the clinical and demographic variables as a predictor of relapse (see Table 2). A comparison of the associated F-to-enter statistics indicates that fast $\beta$ power explained more variance in

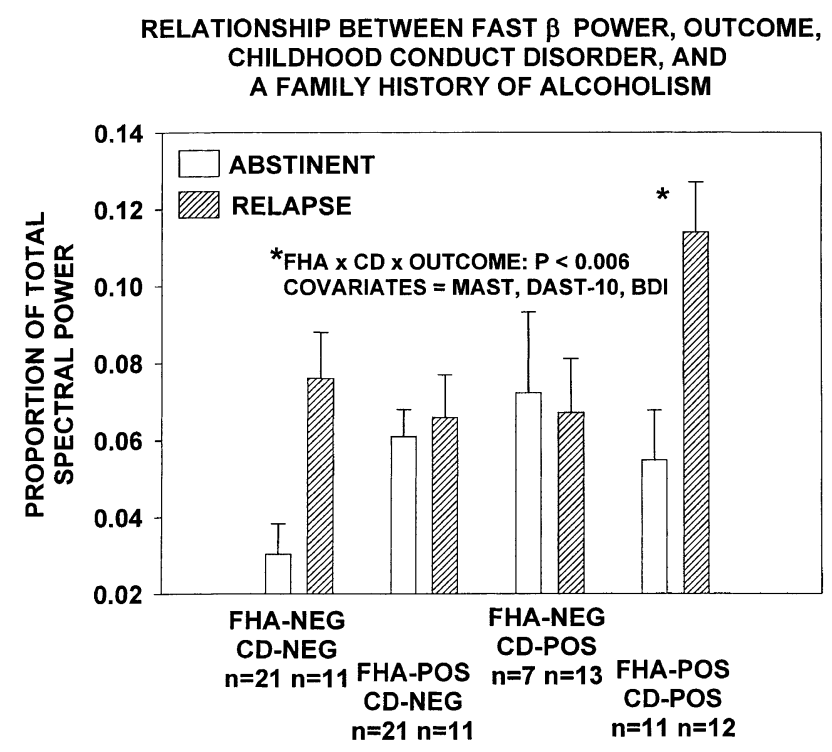

Figure 3. Relative power ( $+1 \mathrm{SE})$ in the fast $\beta$ frequency band as a function of outcome and two premorbid risk factors for substance dependence, viz., childhood Conduct Disorder and paternal alcohol dependence. 
Table 2. Logistic Regression Analysis Results

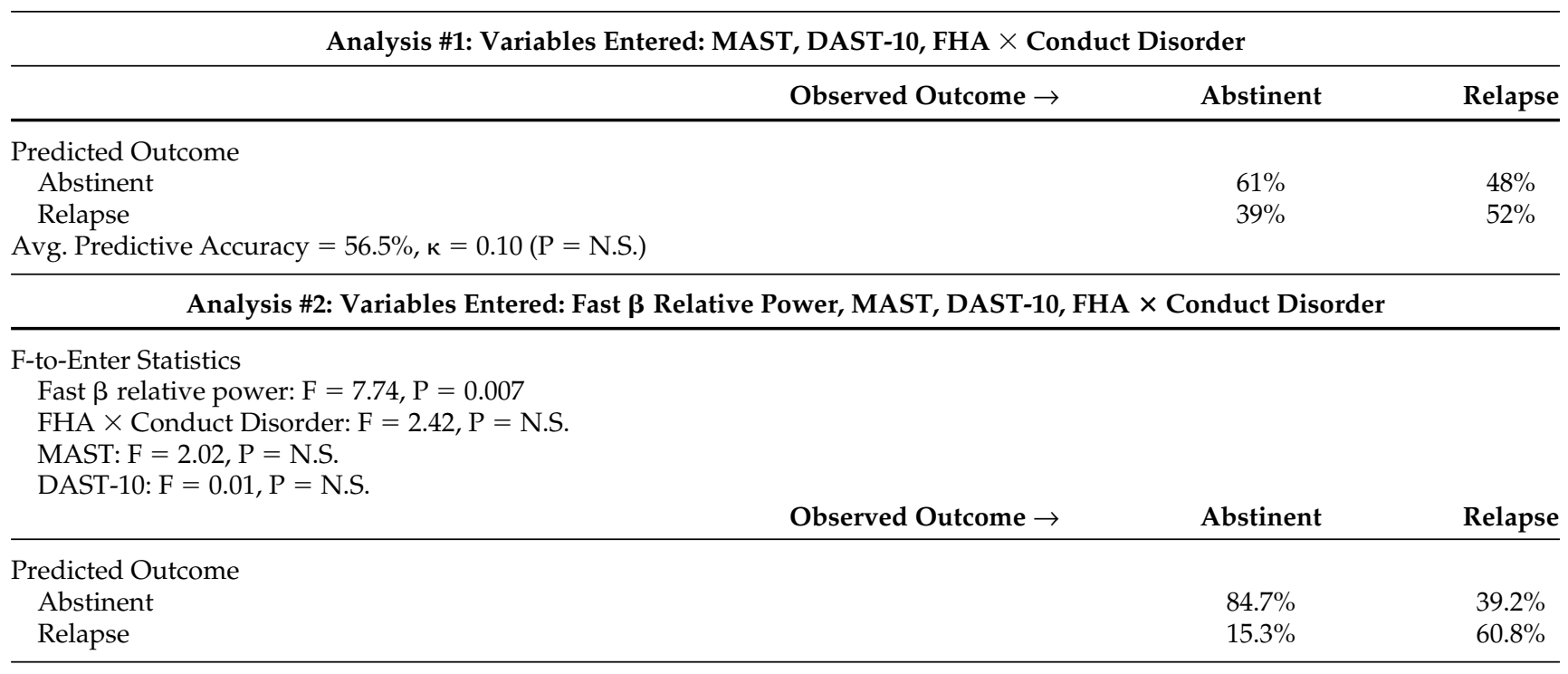

Avg. predictive accuracy $=74.3 \%, \kappa=0.45(p=.001)$; positive predictive value $=0.75$; negative predictive value $=0.73$.

predicting relapse than any of the remaining putative predictors. Relative fast $\beta$ power was, in fact, the only predictor that attained statistical significance within the step-wise model. The best-fitting logistic regression equation was: Outcome $\{0=$ abstinent, $1=$ relapse, cutpoint $=0.5\}=(13.51 *$ Fast $\beta$ Relative Power $)-1.06$.

\section{DISCUSSION}

In summary, the present results, and those reported by others, indicate that the value of EEG fast $\beta$ power for predicting relapse can be generalized across patients with histories of either alcohol, cocaine, cocaine and alcohol, or opioid dependence. Furthermore, the time at which the EEG is recorded does not compromise its predictive value. In both the Winterer et al. (1998) and Bauer (1994) studies, the EEG was recorded from hospitalized patients after one week of abstinence. In the present study, the EEG was recorded from residential treatment program patients after an average of three months of abstinence.

Some readers might question the neural origin of fast $\beta$ power and whether it genuinely reflects brain activity or is merely an artifact of movement and associated electromyographic activity. The careful methods by which the EEG data were screened (see Methods) for the absence of electromyographic artifact argue against the latter alternative. Furthermore, fast $\beta$ power exhibits high heritability (Eischen et al. 1995; Propping et al. 1981) and excellent test-retest reliability (Lund et al. 1995; Nagakubo et al. 1991). Heritability and reliability are not characteristics of an artifact.
A critical reader might likewise be curious about neuropathological mechanisms which mediate the association between fast $\beta$ power and relapse risk. In this regard, it is important to note that current source density analysis techniques employed in the present study (see Figure 2) localized the source of fast $\beta$ activity to deep, anterior regions of the frontal brain. A frontal source for the underlying neuropathology was also suggested by the results of a previous P300 eventrelated potential study (Bauer 1997) of cocaine abusers at lower versus higher risk for relapse. In that study, relapse-prone patients exhibited a specific deficit in the amplitude of the P3a subcomponent. The P3a subcomponent appears to be generated within the orbitofrontal cortex (Clark et al. 2000). Thus, the results of two analyses now suggest that relapse-prone patients exhibit a deficit in the function of a brain region that normally serves to dampen impulsivity and emotionality.

An unresolved question in understanding the neuropathology of relapse risk relates to the frequency band which discriminated the groups. Within the confines of non-quantitative EEG evaluations performed by clinical electroencephalographers, $\beta$ activity is often discounted because it offers no value for localizing specific sources of seizure activity. Furthermore, enhanced $\beta$ activity is rarely detected among patients with diffuse cerebral atrophy secondary to probable Alzheimer's Disease and other forms of dementia (Niedermeyer 1999). It is worth noting, however, that enhanced low voltage, high frequency activity will sometimes presage the larger voltage, low frequency spike and/or wave complexes that define an epileptic event. No one should infer from this statement that the enhanced EEG $\beta$ activity found in relapse-prone patients is equivalent to epileptic activity. 
Rather, it may only reflect a subtle and persistent dysregulation of neural coherence that superficially resembles the abnormal EEG activity detected before seizure onset.

The association demonstrated presently between fast $\beta$ power and two premorbid risk factors-namely, childhood Conduct Disorder and a family history of alcoholism-is not unprecedented in the literature. In 1993, we (Bauer and Hesselbrock 1993) reported that young adult men who possessed both of these risk factors also exhibited a relative enhancement in EEG fast $\beta$ power. Importantly, the young men enrolled in our 1993 study did not meet DSM-IIIR criteria for psychoactive substance dependence. It therefore appears that the abnormal elevation in fast $\beta$ power among patients who later relapse is determined by the conjoint effects of these premorbid risk factors.

The importance and ultimate clinical applicability of the present findings for the management or treatment of substance abuse is tempered by the need to validate our regression equation in an independent and more heterogeneous sample of substance abusers. Yet, the present findings do suggest that EEG assessments could now be useful in treatment matching research or other controlled research endeavors. Future studies should evaluate the question of whether fast $\beta$ power retains its value for predicting relapse among medically-complicated substance abusers, or among substance-abusing patients receiving medications, e.g., benzodiazepines (Bauer 2000), which alter the frequency distribution of the EEG. If specific medications or medical disorders are found to reduce the prognostic significance of fast $\beta$ power, then researchers could either develop a different EEG threshold for detecting future relapse in these specific subpopulations or choose to not apply the predictive power of the EEG in these patients.

One should also consider the importance of the present findings in the context of current restrictions on research and health care budgets, and the efficiencies that may derive from directing prevention and treatment efforts toward individuals at highest risk for relapse. With regard to budget restrictions, for example, it is noteworthy that EEG technology is more practical and affordable than the other neuroimaging technologies, including fMRI, PET, or SPECT. Accordingly, EEG technology can be realistically implemented in large or small treatment settings for either research or clinical indications. Its superiority, relative to clinical or demographic measures, in predicting relapse suggests that it may have value in guiding patients toward more versus less intensive treatments. The inferior spatial resolution of the EEG, relative to the other neuroimaging modalities, can be remedied in part through the use of high density electrode arrays and modern statistical methods of current source localization. Such methods, which were employed in the present study, and dipole localization methods, which were employed in a previous study (Michel et al. 1992), implicate deep, anterior regions of the frontal lobe as the likely generator of EEG fast $\beta$ activity. It is therefore reasonable to hypothesize that the normal function of these regions is altered among patients at high risk for relapse to substance abuse.

\section{ACKNOWLEDGMENTS}

This research was supported in part by U.S. Public Health Service grants R01DA05826, R01DA08598, P50AA03210, and R01MH61346. The author gratefully acknowledges the cooperation of the following treatment centers: Community Substance Abuse Treatment Centers Inc., Blue Ridge Center/ St. Francis Hospital, Alternate Living Center, Clayton House, Coventry House, Connecticut Renaissance, Help Inc., and Open Hearth.

\section{REFERENCES}

Bauer LO (1994): Electroencephalographic and autonomic predictors of relapse in alcohol-dependent patients. Alcohol Clin Exp Res 18:755-760

Bauer LO (1997): Frontal P300 decrements, childhood conduct disorder, family history, and the prediction of relapse among abstinent cocaine abusers. Drug Alcohol Depend 44:1-10

Bauer LO (2000): Electroencephalographic studies of substance use and abuse. In Kaufman MJ (ed), Brain Imaging in Substance Abuse: Research, Clinical, and Forensic Applications. Totowa, NJ, Humana Press, pp 77-112

Bauer LO, Hesselbrock VM (1993): EEG, autonomic, and subjective correlates of the risk for alcoholism. J Stud Alcohol 54:577-589

Beck AT, Ward CH, Mendelson M, Mock J, Erbaugh J (1961): An Inventory for Measuring Depression. Arch Gen Psychiatry 4:561-568

Bobo JK, McIlvain HE, Leed-Kelly A (1998): Depression screening scores during residential drug treatment and risk of drug use after discharge. Psychiatr Serv 49:693-695

Booth BM, Yates WR, Petty F, Brown K (1991): Patient factors predicting early alcohol-related readmissions for alcoholics: Role of alcoholism severity and psychiatric comorbidity. J Stud Alcohol 52:37-43

Brown RA, Monti PM, Meyers MG, Martin RA, Rivinus T, Dubreuil ME, Rohsenow DJ (1998): Depression among cocaine abusers in treatment: Relation to cocaine and alcohol use and treatment outcome. Am J Psychiatry 155:220-225

Bucholz KK, Cadoret R, Cloninger CR, Dinwiddie SH, Hesselbrock VM, Nurnberger JI, Reich T, Schmidt I, Schuckit MA (1994): A new semi-structured psychiatric interview for use in genetic linkage studies: A report on the reliability of the SSAGA. J Stud Alcohol 55:149-158

Clark VP, Fannon S, Lai S, Benson R, Bauer LO (2000): 
Responses to rare visual target and distractor stimuli using event related fMRI. J Neurophysiol 83:3133-3139

Cotton NS (1979): The familial incidence of alcoholism: A review. J Stud Alcohol 40:89-116

Daley DC, Marlatt GA (1997): Relapse prevention. In Lowinson JH, Ruiz P, Millman RB, Langrod JG (eds), Substance Abuse: A Comprehensive Textbook 3rd ed. Baltimore, Williams \& Wilkins Publishers, pp 458-466

Eischen SE, Luckritz JY, Polich J (1995): Spectral analysis of EEG from families. Biol Psychol 41:61-68

Emrick C (1974): A review of psychologically oriented treatment of alcoholism. J Stud Alcohol 35:523-549

Hesselbrock MN (1991): Gender comparison of antisocial personality and depression in alcoholism. J Subst Abuse 3:205-219

Hodgins DC, Elgebaly N, Armstrong S, Dufour M (1999): Implications of depression on outcome from alcohol dependence. A three-year prospective follow-up. Alcohol Clin Exp Res 23:151-157

Hunt W, Barnett L, Branch L (1971): Relapse rates in addiction programs. J Clin Psychol 27:455-456

Kampman KM, Volpicelli JR, McGuinnis DE, Alterman AI, Weinrieb RM, D'Angelo L, Epperson LE (1998): Reliability and validity of the Cocaine Selective Severity Assessment. Addict Behav 23:449-461

Kranzler HR, DelBoca FK, Rounsaville BJ (1996): Comorbid psychiatric diagnosis predicts three-year outcomes in alcoholics: A post-treatment natural history study. J Stud Alcohol 57:619-626

Langenbucher J, Sulesund D, Chun T, Morganstern J (1996): Illness severity and self-efficacy as course predictors of DSM-IV alcohol dependence in a multi-site clinical sample. Addict Behav 21:543-553

Leal J, Ziedonis D, Kosten T (1994): Antisocial personality disorder as a prognostic factor for pharmacotherapy of cocaine dependence. Drug Alcohol Depend 35:31-35

Lund TR, Sponheim SR, Iacono WG, Clementz BA (1995): Internal consistency reliability of resting EEG power spectra in schizophrenic and normal subjects. Psychophysiology 32:66-71

McKay JR, Alterman AI, Rutherford MJ, Cacciola JS, McLellan AT (1999): The relationship of alcohol use to cocaine relapse in cocaine dependent patients in an aftercare study. J Stud Alcohol 60:176-180

McLellan AT, Alterman AI, Metzger DS, Grissom GR, Woody GE, Luborsky L, O'Brien CP (1994): Similarity of outcome predictors across opiate, cocaine, and the alcohol treatments: Role of treatment services. J Consult Clin Psychol 62:1141-1158

McLellan AT, Luborsky L, Woody GE, O’Brien CP (1980): An improved diagnostic evaluation instrument for substance abuse patients (the Addiction Severity Index). J Nerv Ment Dis 168:26-33

Michel CM, Lehmann D, Henggeler B, Brandeis D (1992): Localization of the sources of EEG delta, theta, alpha, and beta frequency bands using the FFT dipole approximation. EEG Clin Neurophysiol 82:38-44

Miller W, Hester R (1986): Inpatient alcoholism treatment: Who benefits? Am Psychol 41:794-805

Nagakubo S, Kumagai N, Kameyama T, Fukuda M, Shirayama Y, Anzai N, Niwa S (1991): Diagnostic reliability and significance of irregular beta patterns. Jpn J Psychiatry Neurol 45:631-640

Niedermeyer E (1999): The normal EEG of the waking adult. In Niedermeyer E, Lopes Da Silva F (eds), Electroencephalography: Basic principles, clinical applications, and related fields, 4th ed. Baltimore, Williams and Wilkins Publishers, pp 149-173

Prichep LS, Alper KR, Kowalik SC, Vaysblat LS, Merkin HA, Tom M, John ER, Rosenthal MS (1999): Prediction of treatment outcome in cocaine dependent males using quantitative EEG. Drug Alcohol Depend 54:35-43

Propping P, Kruger J, Mark N (1981): Genetic disposition to alcoholism: An EEG study in alcoholics and their relatives. Hum Genet 59:51-59

Robins LN (1966): Deviant Children Grown Up. Baltimore, Williams and Wilkins

Rounsaville BJ, Kosten TR, Weissman MM, Kleber HD (1986): Prognostic significance of psychopathology in treated opiate addicts. Arch Gen Psychiatry 43:739-745

Sellman JD, Joyce PR (1996): Does depression predict relapse in the six months following treatment for men with alcohol dependence? Aust N Z J Psychiatry 30:573-578

Selzer ML (1971): The Michigan Alcoholism Screening Test: The quest for a new diagnostic instrument. Am J Psychiatry 127:1653-1658

Shipley WC (1940): A self-administering scale for measuring intellectual impairment and deterioration. J Psychol 9:371-377

Skinner HA (1982): The Drug Abuse Screening Test. Addict Behav 7:363-371

Spielberger DC (1983): State Trait Anxiety Inventory. Palo Alto, CA, Consulting Psychologist Press

Winterer G, Kloppel B, Heinz A, Ziller M, Dufeu P, Schmidt LG, Herrmann WM (1998): Quantitative EEG (QEEG) predicts relapse in patients with chronic alcoholism and points to a frontally-pronounced cerebral disturbance. Psychiatry Res 78:101-113

Woody GE, McLellan AT, Luborsky L, O'Brien C (1985): Sociopathy and psychotherapy outcome. Arch Gen Psychiatry 42:1081-1086

Woody GE, McLellan AT, Luborsky L, O’Brien C, Blaine J, Fox S, Herman L, Beck AT (1984): Severity of psychiatric symptoms as a predictor of benefits from psychotherapy: The Veterans Administration-Penn Study. Am J Psychiatry 141:1172-1177

Ziedonis DM, Kosten TR (1991): Depression as a prognostic factor for pharmacological treatment of cocaine dependence. Psychopharmacol Bull 27:337-343 